\title{
Carcinoma papilar de tiroides en quiste tirogloso*
}

\author{
Drs. JULIÁN ANDRÉS MOLANO G. ${ }^{1}$, GONZALO ROSSEL DE LA M. ${ }^{1}$, \\ MIGUEL GONZÁLEZ P. ${ }^{1}$, INGRID PLASS DEL C. ${ }^{1}$ \\ 1 Departamento de Cirugía Hospital Barros Luco Trudeau. Universidad de Chile. \\ Santiago, Chile.
}

Abstract

\section{Papillary carcinoma in thyroglossal duct cyst}

Background: To report a rare case of papillary carcinoma in thyroglossal duct cyst and discuss its management. Material and Method: A 72 year-old man was clinically diagnosed to have a big thyroglossal duct cyst. Sistrunk's procedure followed by total thyroidectomy on the evidence of papillary cancer in thyroglossal duct cyst. Results: Histopathologic examination revealed a papillary carcinoma in the thyroglossal duct cyst and normallity in the thyroid gland. The patient remained free of disease on follow-up. Conclusion: Most cancers arising in thyroglossal duct cyst are of low risk, and Sistrunk`s procedure with a total thyroidectomy is an adequate treatment for such cancers. tomy.

Key words: Papillary carcinoma, thyroglossal duct cyst carcinoma, Sistrunk's procedure, thyroidec-

\section{Resumen}

Objetivo: Reportar un caso raro de carcinoma papilar en un quiste tirogloso y discutir su manejo. Paciente y Método: Un hombre de 72 años a quien se realizó un diagnóstico clínico de un quiste tirogloso, fue llevado a una cirugía de Sistrunk's y luego una tiroidectomía total ante la evidencia de cáncer papilar en el quiste tirogloso. Resultados: El examen histopatológico reveló un carcinoma papilar de tiroides en el quiste tirogloso y la glándula tiroides fue normal, el paciente permaneció libre de enfermedad en su seguimiento. Conclusiones: La mayoría de los cánceres que surgen en quiste del conducto tirogloso son de bajo riesgo, y el procedimiento Sistrunk’s con una tiroidectomía total es un tratamiento adecuado para este tipo de cáncer. dectomía.

Palabras clave: Carcinoma papilar, carcinoma en quiste tirogloso, procedimiento de Sistrunk's, tiroi-

\section{Introducción}

Los quistes tiroglosos son las anormalidades más comunes de la línea media cervical en relación al desarrollo de la glándula tiroides, la aparición de cáncer tiroideo en estos quistes es una entidad poco común; desde la primera descripción de Bretano en 1911 hasta el presente, sólo 215 pacientes con carcinoma tiroideo en quistes tiroglosos han sido reportados en la literatura ${ }^{1,2}$. Generalmente su diag-

*Recibido el 27 de octubre de 2012 y aceptado para publicación el 2 de diciembre de 2012.

Los autores no refieren conflictos de interés.

Correspondencia: Dr. Julián Andrés Molano G. Avda. Gran Avenida 3204, Santiago, Chile. jamolanog@hotmail.com 


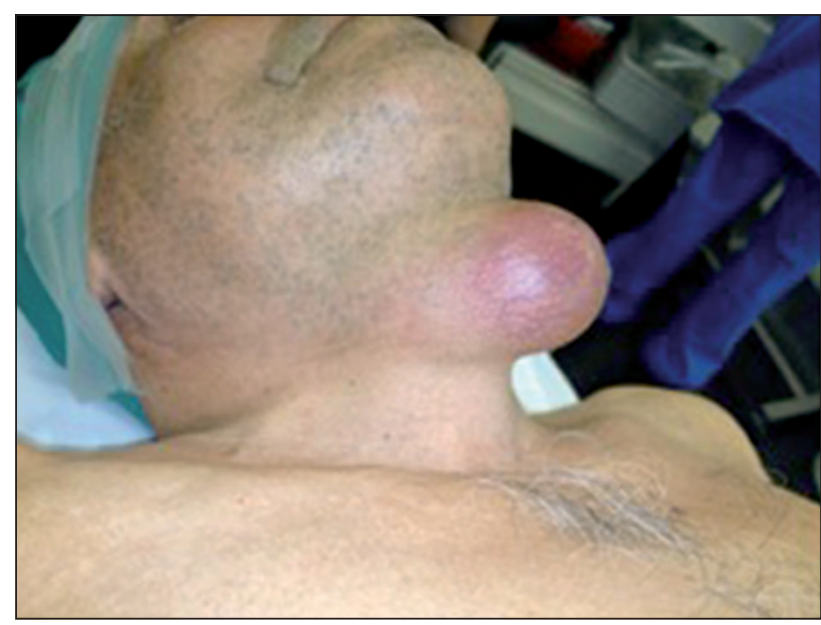

Figura 1. Masa cervical de la línea media correspondiente a quiste tirogloso.

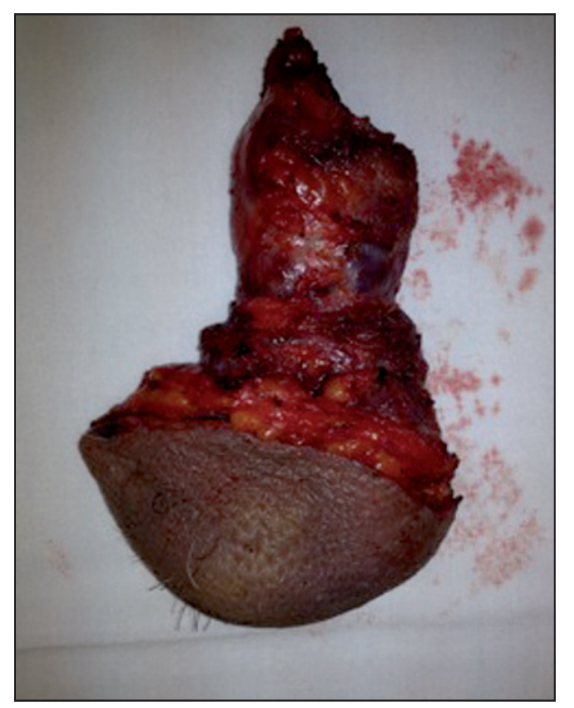

Figura 2. Espécimen quirúrgico la parte superior de la pieza muestra corresponde a la pirámide tiroidea.

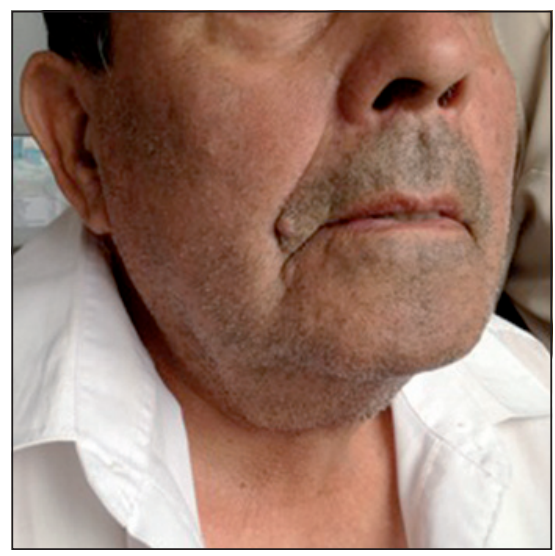

Figura 3. Paciente en posoperatorio 3 meses después. nóstico se realiza de manera sorpresiva no sólo para el paciente si no también para el cirujano. Por lo poco común de esta entidad su manejo suele ser controversial. No existe un consenso claro sobre el manejo de la misma después de una adecuada escisión del quiste. Se han propuesto varias terapias que podrían ser agresivas que podrían tener secuelas a largo plazo, como realizar tiroidectomía total, terapia con yodo radioactivo y terapia con reemplazo hormonal. Dado lo raro de la entidad es difícil que exista una terapia reglada ante el hallazgo de un carcinoma papilar en un quiste tirogloso y depende de la experiencia y criterio médico de cada grupo ${ }^{3}$ (Figura 1).

\section{Tratamiento}

Se realiza una operación de Sistrunk en donde se encuentra una lesión quística infrahioidea que se extendía hasta la región de la pirámide tiroidea (Figura 2).

El reporte de la patología evidencia un quiste tirogloso de $8 \mathrm{~cm}$ y en forma asociada un carcinoma papilar tiroideo bien diferenciado en algunas células tiroideas que tapizan el conducto del quiste tirogloso, no se encuentra permeación vascular o linfática y los márgenes de resección están libres de tumor. Se solicita ecografía tiroidea que muestra un nódulo quístico de $8 \mathrm{~mm}$ en lóbulo derecho. Se decide llevar en un segundo tiempo quirúrgico a una tiroidectomía total con un reporte de patología con una tiroides sana, sin evidencia de malignidad.

El paciente no tuvo complicaciones luego de su cirugía y se mantuvo en controles con reemplazo hormonal tiroideo y no se dio ningún otro manejo complementario con tiroglobulinas negativas hasta 3 meses después de su cirugía (Figura 3).

\section{Discusión}

Los remanentes del conducto tirogloso han sido encontrados alrededor del 7\% de los adultos ${ }^{4}$. Sin embargo, los carcinomas en los quistes del conducto tirogloso son raros y se producen entre un $0,7 \%$ a $1,5 \%$ de todos los quistes del conducto tirogloso ${ }^{5,6}$. Existen algunas especulaciones en la literatura en cuanto al origen de los carcinomas tiroideos bien diferenciados en los quistes del conducto tiroglosos, pero el tejido tiroideo ha sido demostrado histológicamente en dos terceras 
partes de los quistes de los conductos tiroglosos ${ }^{6}, \mathrm{y}$ la transformación maligna es posible al igual que en la glándula tiroides. El diagnóstico de carcinoma en un quiste del conducto tirogloso usualmente es una sorpresa para paciente y médico tratante. Aunque el manejo de los quistes tiroglosos por si solos es sencillo, se convierte en algo controversial cuando en el mismo se hace un hallazgo que generalmente es incidental de un carcinoma de tiroides.

Los carcinomas del conducto tirogloso se presentan más comúnmente hacia la cuarta década de la vida. La mayoría de estos tumores son pequeños y confinados al quiste, aunque unos pocos muestran invasión capsular. Nódulos metastásicos en el cuello se encuentran entre un $7 \%$ a $15 \%$ de los pacientes, pero las metástasis a distancian son muy raras?

Las malignidades deben ser sospechadas en quistes de la línea media cuando se encuentren masas duras adheridas a planos profundos y que se asocian además a evidencia de adenopatías patológicas en cadenas ganglionares cervicales. El procedimiento más adecuado para pacientes con carcinomas del conducto tirogloso es la operación de Sistrunk consistente en la resección del quiste, la porción central del hueso hioides y una capa de tejido al rededor del tracto tirogloso extendiéndose hasta el foramen ciego. La simple escisión del quiste es inadecuada y provee una pobre sobrevida comparada con la operación de Sistrunk.

Se cree por algunos autores que estos tumores y las metástasis de un cáncer tiroideo oculto hacen necesario la realización de una tiroidectomía total como parte del tratamiento definitivo ${ }^{8}$.

Otra razón para realizar la tiroidectomía es la ocurrencia de cáncer concomitante en la glándula tiroides de pacientes adultos con carcinoma en quistes del conducto tirogloso. Además la tiroidectomía realizada con una operación de Sistrunk provee seguridad y tratamiento efectivo para el cáncer además de poder realizar un seguimiento a largo plazo con la realización de tiroglobulinas periódicas o rastreos con yodo radioactivo, considerando las posibilidades de poder generar metástasis con el tiempo. Igualmente se sabe que las complicaciones de una tiroidectomía total en manos expertas tiene una baja tasa de morbilidad.

\section{Conclusiones}

Los carcinomas en quistes del conducto tirogloso son poco comunes, ocurren en aproximadamente el $1 \%$ de todos los quistes del conducto tirogloso. La mayoría de los carcinomas en los quistes tiroglosos son bien diferenciados y en gran porcentaje se diagnostican en forma incidental después de la resección quirúrgica. La operación de Sistrunk realizada en conjunto con una tiroidectomía total en manos expertas provee seguridad en el control del cáncer y permite realizar un adecuado seguimiento con marcadores como la tiroglobulina o con rastreos con radioyodo en busca de posibles recidivas o metástasis posteriores.

\section{Referencias}

1. Brentano H. Struma aberrata lingual mit druzen metastasen. Deutsch Med Wschr 1911;37:665-6.

2. Luna-Ortiz K, Hurtado-López LM, ValderramaLandaeta JL, Ruiz-Vega A. Thyroglossal duct cyst with papillary carcinoma: what must be done? Thyroid 2004;14:363-6.

3. Snehal G Patel, Margarita Escrig, Ashok R Shaha, Bhuvanesh Singh, Jatin P Shah. Management of welldiferentiated thyroid carcinoma presenting within a thyroglosal duct cyst. Journal of Surgical Oncology 2002;79:134-9.

4. Ellis PD, van Nostrand AW: The applied anatomy of thyroglossal tract remnants. Laryngoscope 1977;87:76570.

5. Heshmati HM, Fatourechi V, van Heerden JA, Hay ID, Goellner JR. Thyroglossal duct carcinoma: report of 12 cases. Mayo Clin Proc. 1997;72:315-9.

6. LiVolsi VA, Perzin KH, Savetsky L: Carcinoma arising in median ectopic thyroid including thyroglossal duct tissue. Cancer 1974;34:1303-15.

7. Mazzaferri EL. Thyroid cancer in thyroglossal duct remnants: a diagnostic and therapeutic dilemma. Thyroid 2004;14:335-6.

8. LaRouere MJ, Drake AF, Baker SR, Richter HJ, MagielSki JE. Evaluation and management of a carcinoma aris- ing in a thyroglossal duct cyst. Am J Otolaryngol. $1987 ; 8: 351-5$. 